\title{
The Influence of Soccer Playing Actions on the Recovery Kinetics After a Soccer Match
}

\author{
Mathieu Nedelec, ${ }^{1,2}$ Alan McCall, ${ }^{1,2}$ Chris Carling, ${ }^{2}$ Franck Legall, ${ }^{1,2}$ Serge Berthoin, ${ }^{1}$ \\ AND Gregory Dupont ${ }^{1,2}$ \\ ${ }^{1}$ The University of Lille Nord de France, Lille, France; and ${ }^{2}$ LOSC Lille Metropolitan Football Club, Camphin-en-Pévele, \\ France
}

\begin{abstract}
This study examined the relationship between the frequency of playing actions performed during a soccer match and the recovery kinetics after the match. Time motion analyses were performed on 10 professional soccer players during 4 competitive matches (14 observations) to determine the number of playing actions completed by players. Subjective ratings, creatine kinase, and physical tests (countermovement jump [CMJ], isometric maximum voluntary contraction of the hamstrings, 6-second sprint on a nonmotorized treadmill) were performed before the match and 24 hours, 48 hours, and 72 hours after the match. During the 72-hour recovery period, CMJ, isometric strength of the hamstring muscles, and peak sprint speed significantly ( $p \# 0.05)$ decreased, whereas muscle soreness increased ( $p \#$ 0.05). Significant correlations were observed between the increase in muscle soreness and number of short sprints $(, 5 \mathrm{~m})$ performed at 48 hours $(r=0.74$; confidence interval [Cl], 0.35-0.91; $p, 0.01)$ and 72 hours $(r=0.57 ; \mathrm{Cl}$, $0.05-0.84 ; p \# 0.05)$ after match play. A significant relationship ( $r=20.55 ; \mathrm{Cl}, 20.84$ to 20.03; $p$ \# 0.05) was also observed between CMJ performance decrement at 24 hours and the number of hard changes in direction performed. Soccer match play resulted in significant neuromuscular fatigue for up to 72 hours after match and was dependent on the number of sprints and hard changes in direction performed during the match. Time motion analysis data currently used during a soccer match should quantify hard changes in direction, acceleration and deceleration phases to enable better estimations of postmatch fatigue.
\end{abstract}

KEY WORDS motion analysis, performance, football, fatigue

Address correspondence to Mathieu Nedelec, matnedelec@orange.fr.

\section{INTRODUCTION}

I $\mathrm{n}$ professional soccer, the number of competitive matches played per season is high, and players are frequently required to play consecutive matches interspersed by 3-day intervals. Participation in a soccer match leads to acute fatigue characterized by a decline in physical performance over the following hours and days $(3,4,20)$. During congested schedules, estimating matchrelated fatigue is necessary to manage recovery strategies and training loads because inappropriate training loads within a between-match microcycle can negatively impact neuromuscular and perceptual recovery (26). Unfortunately, the wearing of electronic monitoring devices (e.g., heart rate monitor, global positioning system) is not allowed during Union of European Football Associations official soccer competitions (29). Therefore, indirect measures of work load such as total distance covered and that run at different intensities using automated computer-aided time motion analyses are currently the only means to assess player work rate during official match play (9). These physical data are based on the assumption that they reflect the volume of work performed and intensity of the match, and consequently the level of fatigue experienced by players. However, acceleration and deceleration running phases (29), and contacts with opposing players, jumps, and tackles (37) should also be included in the analysis of players' physical efforts. These common match events have been shown to induce muscle damage $(10,19,27)$ and their frequency might affect the time to fully recover after competition. For example, Young et al. (39) reported that acceleration and deceleration actions performed in Australian rules football competition were large contributors to muscle damage estimated by determining creatine kinase $(\mathrm{CK})$ concentrations 24 hours after match. However, to our knowledge, the influence of such actions on postmatch recovery kinetics in soccer has not been reported.

The purpose of this study was to investigate the relationship between the recovery kinetics after a soccer match and the number of soccer specific playing actions performed during play. We hypothesized that the number of soccer specific playing actions performed during a match is related to the time to fully recover from that match. 


\section{Methods}

\section{Experimental Approach to the Problem}

Time motion analyses were performed from video recordings for outfield professional soccer players playing a minimum of 75 minutes during 4 home competitive matches (between January and May 2012) to establish the number of playing actions completed by players. During the 72 hour-period after each match, the players provided subjective ratings and performed physical tests, whereas $\mathrm{CK}$ concentrations were used to evaluate muscle damage. The various relationships between the number of playing actions performed during the match and changes in subjective ratings and physical tests throughout recovery were examined to provide insight into the influence of these actions on the recovery kinetics after a soccer match.

All visits to the laboratory occurred at the same time of the day throughout the protocol (between 9:00 and 10:00 AM). After a familiarization session, 2 preliminary sessions were performed to determine reference values. To limit dietary influences on test results, players were asked to follow standardized nutritional guidelines (quantity and content for food and drink) throughout the data collection period. During and immediately after the match, players drank a standardized volume of sports drink containing $6 \%$ of carbohydrate (Gatorade; PepsiCo, Purchase, NY, USA). Players were requested not to use any different recovery treatments (cold bath, massage, compression garments) throughout the postmatch data collection period, which may have affected their recovery. As players belonged to a professional team, the tests were performed before their usual training sessions. The content of these training sessions is presented in Table 1. The training loads for each training session were recorded using methods described by Foster et al. (14). This method calculates a total training load (arbitrary units) by multiplying the session-rating of perceived exertion (RPE) by the session time.

\section{Subjects}

Ten professional outfield soccer players (age, 21.863 .2 years; range: 17.5-29.0 years; height, $178.165 .4 \mathrm{~cm}$; body mass,
$76.567 .6 \mathrm{~kg}$; and body fat, $9.361 .7 \%$ ) representing common playing positions ( 2 fullbacks, 2 central defenders, 3 midfielders, 2 wingers, and 1 forward) participated in the study. Players participated in 1 match and 7 training sessions per week (volume, 11-14 hours) throughout the season. During the 4 competitive matches analyzed, 2 players (a central defender and a midfielder) were followed during 2 different matches and, 1 fullback was followed during 3 different matches. As a result, a total of 14 individual recordings were made. All players completed the full match (90 minutes), except the forward (78 minutes) and 1 midfi lder (86 minutes) because of substitutions made for tactical reasons. Before the study, players completed a medical examination to check that they were not injured or ill, nor taking any medication or drug. Body fat was estimated from 4-site skinfold measurements in triplicate by means of a Lange caliper (Cambridge Scientifi Instruments, Cambridge, MD, USA) (11). All players provided written informed consent in accordance with the ethical committee on biomedical research "Comité de Protection des Personnes Nord Ouest IV" and parental informed consent was also obtained for one subject less than 18 years of age. This study was made with the agreement of the ethical committee on biomedical research "Comité de Protection des Personnes Nord Ouest IV" and the standards set by the Declaration of Helsinki.

\section{Procedures}

Video Match Analysis. Time motion analysis is a useful method to indirectly quantify the physical demands of individual players during match play $(2,6,23)$. The reliability of this method has been reported for locomotive movements (coefficient of variation [CV], 1-5\%) (23) and nonlocomotive movements (kappa $=0.79)(7)$. Each match was videofilmed by the same professional cameraman. The camera (DCR-SR57, Sony, Minato, Tokyo, Japan) was positioned at the side of the pitch, at the level of the midfield line, at a height of about $10 \mathrm{~m}$ and at a distance of $20-30 \mathrm{~m}$ from the touchline. The video footage was later replayed on a monitor for computerized coding of the number of playing actions completed by players. All match recordings included in this

TABLE 1. General description of training content and daily training load (in arbitrary units; mean $6 S D$ ) during the 72-hour period after the competitive soccer match.*

\begin{tabular}{|c|c|c|c|}
\hline & $24 \mathrm{~h}$ Postmatch & $48 \mathrm{~h}$ Postmatch & $72 \mathrm{~h}$ Postmatch \\
\hline $\begin{array}{l}\text { Testing } \\
\text { Training AM }\end{array}$ & $\begin{array}{l}\text { Testing } \\
\text { Nil }\end{array}$ & $\begin{array}{l}\text { Testing } \\
\text { Strength training (75 min) or team training } \\
\quad(75-105 \mathrm{~min}) 2756129\end{array}$ & Testing \\
\hline Training PM & $\begin{array}{l}\text { Upper-body strength training } \\
\text { (50-70 min) } 226689\end{array}$ & $\begin{array}{l}\text { Team training (70-90 min) or strength training } \\
\text { ( } 75 \mathrm{~min}) 113696\end{array}$ & \\
\hline
\end{tabular}

*Testing = subjective ratings, creatine kinase, and physical tests; Team training = attack and defensive patterns, match plans, and general skills; Strength training = lat pull down, push-ups, bench throw, biceps curl, dips, leg press, squats, steps-ups, snatch, and clean-and-jerk. 
study were analyzed by the same experienced observer (2). The video was paused after every event that occurred, and the playing action was notated on paper. Eight soccerspecific playing actions were recorded (Table 2) (31).

Physical Tests. Before the physical performance tests, players performed a standardized warm-up of 10 minutes at $100 \mathrm{~W}$ on a cycle ergometer. Players performed 2 countermovement jumps (CMJ) on a force platform (Kistler AG, Winterhur, Switzerland) with the best jump recorded. The force signal was sampled at $1,000 \mathrm{~Hz}$. Players were instructed to jump explosively upwards immediately after descending to a self-selected depth keeping hands on the hips. Previous work in our laboratory examining the interday test-retest reliability for CMJ among the same population found that this measure is reliable (typical error [TE], $1.5 \mathrm{~cm}$; intraclass correlation coefficient [ICC], 0.95; CV, 3.9\%).

Players performed 1 isometric maximum voluntary contraction (MVC) of the hamstring muscles for the dominant (i.e., kicking leg) and nondominant leg according to the methodology proposed by Schache et al. (34). Players were positioned with knee flexed to 908 and then to 1508 (full extension: 1808). A goniometer (Lafayette Instrument Company, Lafayette, IN, USA) was used to set the angles. The heel of the tested leg rested on a firm plinth of adjustable height, the other leg rested below the plinth (34). The cuff of a sphygmomanometer (Omron Healthcare Co., Ltd, Kyoto, Japan) was preinflated to $10 \mathrm{~mm} \mathrm{Hg}$ and placed under the heel of the tested leg. Players were verbally encouraged to push the heel into the cuff as hard as possible by attempting to flex the knee against the plinth without lifting their buttocks off the ground. The contraction was held for 3 seconds, and the peak pressure was recorded. The process was performed at both angles and for the dominant and nondominant leg, with contractions separated by 60 -second rest periods. The interday test-retest reliability of the methodology was assessed before experimentation with players involved in the study. The measure was proven to be reliable (5) with TE, ICC, and CV ranging from 16 to $21 \mathrm{~mm} \mathrm{Hg}, 0.80-0.88$, and $5.4-7.3 \%$, respectively, according to the tested leg and angle. The validity of the methodology was also assessed before experimentation using 13 players belonging to the present professional team. There was a very high correlation $(r=$ $0.81 ; 95 \%$ confidence intervals $(\mathrm{CI})=0.48-0.94 ; p=0.001)$ between the isometric peak strength of the hamstring muscles with a 908 knee extension measured by the sphygmomanometer and a dynamometer (Con-Trex, Duebendorf, Switzerland), and a high correlation ( $r=0.53$; CI $=20.03$ to 0.84 ; $p=0.06$ ) between the 2 measures with a 1508 knee extension.

Players completed one 6-second sprint on a nonmotorized treadmill (NMT, Woodway Force 3.0, Waukesha, USA) from a standardized start position (i.e., standing start with both hands on the handles). The mean power output (MPO), mean speed (MS), and peak speed (PS) were recorded. Previous work in our laboratory with the same population found very high interday test-retest reliability for MPO, MS, and PS (TE: $90 \mathrm{~W}, 0.2 \mathrm{~m} \$ \mathrm{~s}^{21}$ and $0.2 \mathrm{~m} \$ \mathrm{~s}^{21}$, respectively; ICC: $0.87,0.89$, and 0.88 , respectively; CV: 3.1 , 2.6 , and $2.2 \%$, respectively). The nonmotorized treadmill (NMT; Woodway Force 3.0) was calibrated before each test. Treadmill belt speed, distance, and horizontal forces were collected at a sampling rate of $100 \mathrm{~Hz}$ through the XPV7 PCB interface (Fitness Technology, Adelaide, Australia) and analyzed with the Force 3.0 Software (Innervations Software, Joondalup, Australia).

Creatine Kinase. A pinprick on the finger allowed $32 \mathrm{ml}$ of capillary whole blood to be drawn, which was placed on a measurement strip and analyzed by means of a Reflotron (Roche Diagnostics, Grenzacherstrasse, Switzerland). It was

TABLE 2. List of playing actions recorded during the match.

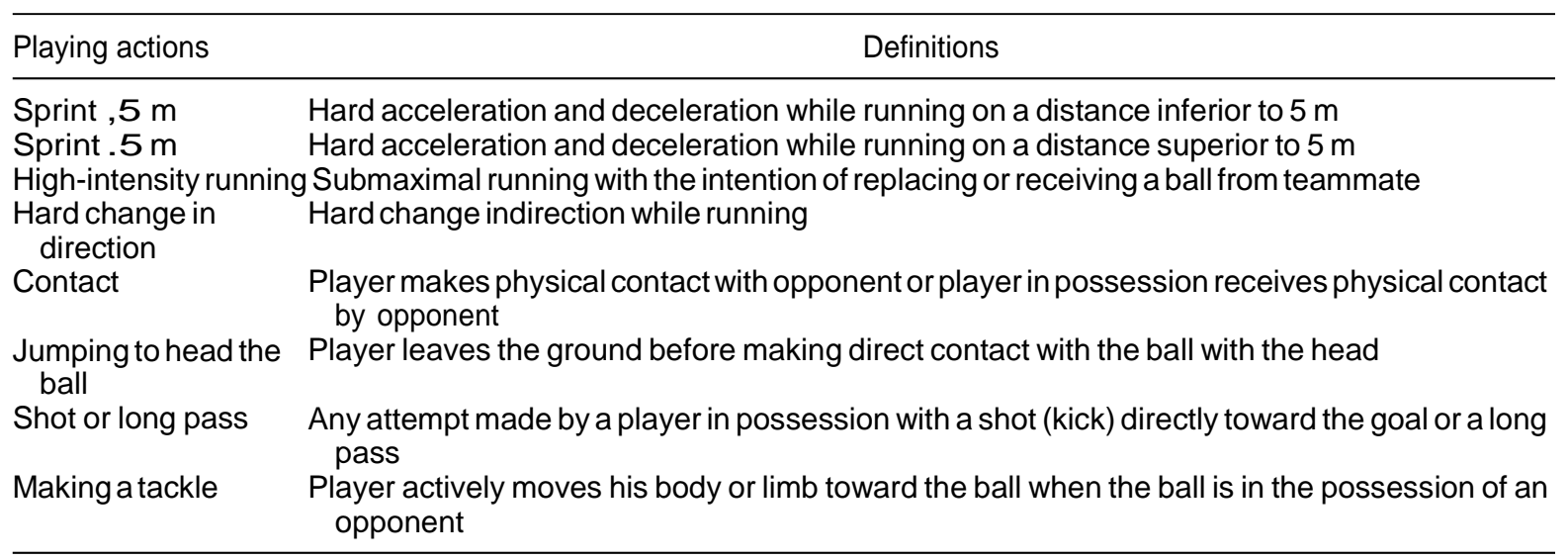


assumed that the CK analyzed emanated from muscle damage rather than from cardiac or brain damage. The Reflotron was calibrated according to the recommendations of the manufacturer, and analysis took place in a controlled laboratory environment. Because of technical problems, only 7 players were analyzed for CK during the postmatch data collection period. Given this low sample size, the correlations between changes in $\mathrm{CK}$ and the number of playing actions completed during the match were not investigated.

Subjective Ratings. Players were required to rate their quality of sleep, fatigue, muscle soreness, and stress using a scale from 1 to 7 points (16). Perceived recovery was rated using the total quality recovery (TQR) scale, encompassing the anchor points 6 (not recovered at all) to 20 (completely recovered). The TQR was conceptualized by Kenttä and Hassmén (21) and has been used in previous studies as an indicator of athletes' perceived recovery $(22,30)$.

\section{Statistical Analyses}

Data are presented as mean 6 SD. The CV, ICC, 95\% confidence intervals (CIs), and TE were calculated according to Hopkins (18). The normality distribution of the data was checked using the Shapiro-Wilk test. The effect of recovery time on the dependent variables-CMJ, MVC, MPO, MS, PS,
$\mathrm{CK}$, and subjective ratings — was analyzed using a 1-way analysis of variance for repeated measures. Bonferroni post hoc was then applied when the significant $F$ value was found. Effect size data were calculated and were assessed using the following criteria: , $0.2=$ trivial, $0.2-0.6=$ small, $0.6-1.2=$ moderate, $1.2-2.0=$ large, and $.2 .0=$ very large (17). The correlations between changes in CMJ, MVC, MPO, MS, PS, subjective ratings, and the number of playing actions completed during the match (expressed in number per minute) were analyzed using the Pearson product-moment correlation coefficient $(r)$. Statistical significance was set at $p$ \# 0.05.

\section{Results}

\section{Playing Actions}

Players performed 16.167 .5 sprints , $5 \mathrm{~m}$ (range, 1-28), 11.265 .8 sprints $.5 \mathrm{~m}$ (range, 2-22), 25.169 .6 highintensity runs (range, 9-43), 11.965 .2 hard changes in direction (range, 2-18), 14.366 .0 contacts (range, 2-27), 10.465 .4 jumps to head the ball (range, 3-19), 8.464 .1 shots or long passes (range, 3-16), and 0.961 .0 tackles (range, $0-3$ ) for a total of 98.5618 .6 playing actions during the match (1.11 60.21 playing action per minute). The lowest (61) and highest (132) number of playing actions were performed by a central defender and a midfielder, respectively.

TABLE 3. Recovery time course of subjective ratings, creatine kinase, and physical tests after competitive soccer match and magnitude of the change (effect size).*

\begin{tabular}{|c|c|c|c|c|c|c|c|}
\hline Time (h after the match) & Reference & +24 & $\begin{array}{c}\text { Effect } \\
\text { size }\end{array}$ & +48 & $\begin{array}{c}\text { Effect } \\
\text { size }\end{array}$ & +72 & $\begin{array}{c}\text { Effect } \\
\text { size }\end{array}$ \\
\hline $\mathrm{CMJ}(\mathrm{cm})$ & 39.962 .2 & $36.962 .9+$ & 1.22 & $37.363 .4 \dagger$ & 1.03 & $37.462 .4 \dagger$ & 1.13 \\
\hline MVC $(\mathrm{mm} \mathrm{Hg}) 908$ dominant & 372641 & $343642 \dagger$ & 0.77 & $347649 z$ & 0.69 & $351644 z$ & 0.62 \\
\hline $\begin{array}{l}\text { MVC }(\mathrm{mm} \mathrm{Hg}) 908 \\
\text { Nondominant }\end{array}$ & 365635 & $342639 z$ & 0.67 & $333638 z$ & 0.96 & 343641 & 0.53 \\
\hline $\begin{array}{l}\text { MVC }(\mathrm{mm} \mathrm{Hg}) 1508 \\
\text { dominant }\end{array}$ & 338633 & $306634 z$ & 1.08 & $312650 \S$ & 0.77 & $310637 z$ & 1.08 \\
\hline $\begin{array}{l}\text { MVC }(\mathrm{mm} \mathrm{Hg}) 1508 \\
\text { Nondominant }\end{array}$ & 333640 & $300640 z$ & 0.99 & $304652 z$ & 0.72 & $308648 z$ & 0.70 \\
\hline MPO (W) & 25536289 & 25286263 & 0.10 & 25046241 & 0.30 & 25206237 & 0.16 \\
\hline $\mathrm{MS}\left(\mathrm{m} \$ \mathrm{~s}^{21}\right)$ & 4.9860 .37 & 4.9460 .34 & 0.15 & 4.8760 .35 & 0.46 & 4.8960 .34 & 0.28 \\
\hline $\mathrm{PS}\left(\mathrm{m} \$ \mathrm{~s}^{21}\right)$ & 5.8660 .30 & $5.6460 .26 \dagger$ & 0.80 & $5.5760 .28 \dagger$ & 1.13 & $5.5860 .29 \dagger$ & 1.02 \\
\hline $\mathrm{CK}\left(U \$ \mathrm{~L}^{21}\right)(N=7)$ & 2486105 & $7276235 \dagger$ & 3.17 & $5086210 z$ & 1.88 & $4706204 \S$ & 1.90 \\
\hline Sleep (au) & 2.461 .3 & 3.361 .3 & 1.08 & 2.661 .3 & 0.36 & 2.661 .3 & 0.33 \\
\hline Fatigue (au) & 2.761 .1 & $4.161 .1 \dagger$ & 2.01 & 3.361 .2 & 1.93 & 3.160 .8 & 0.42 \\
\hline Stress (au) & 1.961 .1 & 2.461 .7 & 0.62 & 2.060 .9 & 0.13 & 1.960 .9 & 0.09 \\
\hline Muscle soreness (au) & 2.160 .9 & $3.661 .2 \dagger$ & 2.13 & $3.861 .3 \dagger$ & 2.34 & $3.561 .5 z$ & 1.50 \\
\hline TQR (au) & 16.162 .5 & $13.661 .7 z$ & 2.93 & 15.161 .9 & 1.10 & 15.361 .6 & 0.53 \\
\hline
\end{tabular}

*au = arbitrary units; $\mathrm{CMJ}=$ countermovement jump; $\mathrm{CK}=$ creatine kinase $; \mathrm{MPO}=$ mean power output; $\mathrm{MS}=$ mean speed; $\mathrm{MVC}=$ isometric maximum voluntary contraction of the hamstring muscles with a 90 and 1508 knee extension for the dominant and nondominant leg; PS = peak speed; TQR = total quality recovery. Magnitudes of effect sizes are assessed using the following criteria: , $0.2=$ trivial, $0.2-0.6=$ small, $0.6-1.2=$ moderate, $1.2-2.0=$ large , and $.2 .0=$ very large .

†Significantly different from reference values $(p, 0.001)$.

zSignificantly different from reference values $(p, 0.01)$.

$\S$ Significantly different from reference values $(p \# 0.05)$ 
Recovery Kinetics for Subjective Ratings, Creatine Kinase, and Physical Tests After the Match

The recovery time course of subjective ratings, CK, and physical tests after soccer match play are presented in Table 3.

\section{Correlations Between Subjective Ratings and Physical Tests and Playing Actions}

Mean power output and MS during 6-second sprint, sleep and stress showed no change from reference values throughout the protocol (Table 3). Consequently, only the correlations between changes in CMJ, MVC, PS, fatigue, muscle soreness, TQR, and playing actions completed during the match were assessed. Significant correlations were observed between the increase in muscle soreness and the number of sprints , $5 \mathrm{~m}$ performed during the match at 48 hours $(r=$ 0.74 ; CI, 0.35-0.91; $p, 0.01)$ and 72 hours $(r=0.57$; CI, $0.05-0.84 ; p \# 0.05)$. A significant relationship $(r=20.55$; CI, 20.84 to 20.03; $p$ \# 0.05) was established between the decrement in CMJ performance at 24 hours and the number of hard changes in direction performed during the match. Significant correlations were found between the decrement in MVC with a 908 knee extension at 48 hours for the dominant leg and the number of tackles ( $r=20.68$; CI, 20.90 to 20.21; $p=0.01$ ), as well as between the decrement in MVC with a 1508 knee extension at 24 hours and the number of tackles made ( $r=20.65$; CI, 20.88 to 20.18; $p=0.01$ ).

\section{Discussion}

The purpose of this study was to investigate the relationship between the recovery kinetics after a soccer match and the number of playing actions performed during that match. We hypothesized that soccer-specific playing actions performed during the match were associated with the time to fully recover from that match. Our results suggest that the soccer match effectively induced neuromuscular fatigue.

In this study, CMJ performance was significantly impaired up to 72 hours postmatch, which is consistent with results obtained with elite female soccer players (3), second division male soccer players (25), professional rugby league players (26), and elite female handball players (33). These findings are in line with previous studies that have shown the stretchshortening cycle recruitment to be strongly implicated with exercise fatigue (28) and because soccer match play involves many stretch-shortening cycle actions, CMJ performance is consequently a useful recovery marker.

In this study, PS achieved during 6-second sprint was more sensible to fatigue than MPO and MS during the sprint. Mean power output and MS were unaffected throughout the recovery process, which is consistent with findings reported by Andersson et al. (3). These authors found that $20-\mathrm{m}$ sprint times measured with photocells returned to baseline values 5 hours after an elite soccer match. Conversely, in this study, PS was impaired throughout the recovery process, which corroborates findings from Slattery et al. (35) who showed that 4 days of high training loads reduced peak sprint speeds $(p, 0.001)$ achieved during a simulated team sport match performance on a nonmotorized treadmill. Fatigue involves changes in maximal force, maximal shortening velocity, and the curvature of the force-velocity relationship with different underlying mechanisms (1). Peak speed impairment during maximal exercise such as sprinting may be explained by a reduction in voluntary activation. A 48-hour reduction in voluntary activation was observed after an elite soccer match with a significant relationship $(r=20.63 ; p, 0.01)$ between sprint performance decrement and maximal voluntary activation immediately after the match (32). Moreover, the reduction in voluntary activation may be because of inhibition caused by muscle soreness (8). The significant relationship ( $r=20.61 ; p$ \# 0.05) we observed between PS decrement and muscle soreness increase 24 hours after the match supports this hypothesis.

Impaired maximal voluntary strength after a soccer match has previously been reported but only in the dominant leg $(3,25,38)$. To our knowledge, this study is the first to assess the recovery of maximal voluntary strength in both limbs after a soccer match. Here, the nondominant leg recovered quicker than the dominant leg, which may be linked to a higher frequency of technical actions performed using the dominant leg (e.g., shot and long pass). Isometric strength of the hamstring muscles with a 908 knee extension was still impaired by $6.765 .7 \%$ at 72 hours for the dominant leg, whereas it was not significant for the nondominant leg. Isometric strength of the hamstring muscles with a 1508 knee extension was impaired to a greater extent at 72 hours for the dominant leg than the nondominant leg. This result may partly explain why a greater number of injuries (50 vs. $37 \% ; p, 0.01)$ are sustained to the players' dominant side compared with the nondominant side in soccer (15). It has previously been suggested that the predominance of injuries to the dominant side is due to that side being more commonly involved when tackling or being tackled (12). We found significant correlations between the decrement in isometric strength of the hamstring muscles with a 908 knee extension at 48 hours for the dominant leg and the number of tackles, as well as at 1508 knee extension at 24 hours also for the dominant leg. The low frequency of tackles performed by players in this study (range, 0-3) prompts us to be cautious in the interpretation of these relationships. Future larger-scale studies are required to confirm differences in the recovery kinetics between the dominant and nondominant leg.

Total playing actions performed during the match tended to correlate with decrements in $\mathrm{CMJ}$ performance $(r=$ 20.50; $p=0.07$ ) and MVC of the hamstring muscles with a 908 knee extension for the dominant leg $(r=20.52 ; p=$ 0.06) 24 hours after the match. Thereafter, no relationship could be observed throughout the recovery period. Similarly, McLellan and Lovell (27) found a relationship between playing actions performed during rugby match play (i.e., impacts associated with collisions) and neuromuscular responses during a CMJ 30 minutes and 24 hours postmatch but not 
later during the 5-day period after the match. The influence of playing actions performed during the match on neuromuscular responses may be short lasting and observed only in the first hours of recovery rather than being persistent throughout the entire recovery period. Several factors operate during the recovery process and have the potential to alter a relationship established in the first hours of recovery. In this respect, future studies are required to identify the potential impact of intrinsic factors such as age (13) or muscle fiber typology (24) to explain interindividual differences in recovery potential among players in the same team. Identifying these intrinsic factors is a prelude to the development of individualized recovery protocols. Finally, it should not be excluded that players experienced different physical stress during the same team training session. To counteract these limitations, future studies could investigate the relationship between the recovery kinetics and the number of playing actions performed during several matches played by the same player (i.e., intraindividual differences in recovery kinetics).

A significant relationship was established between the decrement in CMJ performance at 24 hours and the number of hard changes in direction performed during the match. Concomitantly, the relationship between the decrement in CMJ performance and the number of sprints ,5 m approached significance $(r=20.47 ; p=0.09)$. Indeed, changes in direction are associated with accelerations and decelerations, which are particularly damaging to the muscles involved. Eccentric muscle contractions during these activities are considerable and may explain the marked increase in markers of muscle damage observed after shuttle running test (36) and CMJ impairment after such test (25). Significant correlations were also found between the increase in muscle soreness and the number of sprints $(, 5 \mathrm{~m})$ performed during the match at 48 hours and 72 hours. Short sprints are associated with a rapid deceleration leading to muscle soreness during the following days (19).

A limitation of this study is that the video match analysis only included the frequency of playing actions performed during the match and not the intensity. Future studies may use micro technology, including Global Positioning System (GPS) and accelerometers during friendly matches to examine the influence of the intensity of playing actions on the recovery kinetics following match play as has been demonstrated in rugby for impacts and collisions (27); and in Australian rules football for accelerations and decelerations (39).

\section{Practical Applications}

This study revealed that CMJ performance and peak sprint speed seem to be the most sensible variables for monitoring postmatch neuromuscular status among the variables tested in professional soccer players. The findings of this study also suggest that playing actions commonly performed during a soccer match such as hard changes in direction and acceleration and deceleration phases are associated with
Significant neuromuscular fatigue that remains evident for up to 72 hours. Traditional time motion analysis data (i.e., total distance covered and that run at different intensities) currently used during a soccer match should integrate additional hard changes in direction and acceleration and deceleration phases. Determining both the demands of competition and sensible variables for monitoring recovery can assist coaches and sport scientists to adjust the training load after a match.

\section{ACKNOWLEDGMents}

The authors gratefully acknowledge Lille OSC, the players and coaches for their co-operation, Benoit Delaval, Quentin Robert, Jonathan Catalano, Dr. François Drouard, Damien Lewandowski, and Benoit Bernard for their assistance during the experimentation. The authors have no conflicts of interest that are directly relevant to the content of this article. No financial support was provided for this study. Results of this study do not constitute endorsement of the product by the authors or the National Strength and Conditioning Association.

\section{REFERENCES}

1. Allen, DG, Lamb, GD, and Westerblad, H. Skeletal muscle fatigue: Cellular mechanisms. Physiol Rev 88: 287-332, 2008.

2. Andersson, H, Ekblom, B, and Krustrup, P. Elite football on artificial turf versus natural grass: Movement patterns, technical standards, and player impressions. J Sports Sci 26: 113-122, 2008.

3. Andersson, H, Raastad, T, Nilsson, J, Paulsen, G, Garthe, I, and Kadi, F. Neuromuscular fatigue and recovery in elite female soccer: effects of active recovery. Med Sci Sports Exerc 40: 372-380, 2008.

4. Ascensão, A, Rebelo, A, Oliveira, E, Marques, F, Pereira, L, and Magalhães, J. Biochemical impact of a soccer match-analysis of oxidative stress and muscle damage markers throughout recovery. Clin Biochem 41: 841-851, 2008.

5. Atkinson, G, Nevill, AM, and Edwards, B. What is an acceptable amount of measurement error? The application of meaningful "analytical goals" to the reliability of sports science measurements made on a ratio scale. J Sports Sci 17: 18, 1999.

6. Bangsbo, J, Nørregaard, L, and Thorsø, F. Activity profile of competition soccer. Can J Sport Sci 16: 110-116, 1991.

7. Bloomfield, J, Polman, RCJ, and O'Donoghue, PG. Reliability of the Bloomfield Movement Classification. Int J Perf Anal Sport 7: 20-27, 2007.

8. Byrne, C, Twist, C, and Eston, R. Neuromuscular function after exercise-induced muscle damage: Theoretical and applied implications. Sports Med 34: 49-69, 2004.

9. Carling, C, Bloomfield, J, Nelsen, L, and Reilly, T. The role of motion analysis in elite soccer: Contemporary performance measurement techniques and work rate data. Sports Med 38: 839$862,2008$.

10. Chatzinikolaou, A, Fatouros, IG, Gourgoulis, V, Avloniti, A, Jamurtas, AZ, Nikolaidis, MG, Douroudos, I, Michailidis, Y, Beneka, A, Malliou, P, Tofas, T, Georgiadis, I, Mandalidis, D, and Taxildaris, K. Time course of changes in performance and inflammatory responses after acute plyometric exercise. J Strength Cond Res 24: 1389-1398, 2010.

11. Durnin, JVand Womersley, J. Body fat assessed from total body density and its estimation from skinfold thickness: Measurements on 481 men and women aged from 16 to 72 years. Br J Nutr 32: 77-97, 1974.

12. Ekstrand, J. Soccer Injuries and Their Prevention. Linkoping University Medical Dissertations. Linkoping, Sweden: Linkoping University, 1982. 
13. Fell, J and Williams, D. The effect of aging on skeletal-muscle recovery from exercise: Possible implications for aging athletes. JAging Phys Act 16: 97-115, 2008.

14. Foster, C, Florhaug, JA, Franklin, J, Gottschall, L, Hrovatin, LA, Parker, S, Doleshal, P, and Dodge, C. A new approach to monitoring exercise training. J Strength Cond Res 15: 109-115, 2001.

15. Hawkins, RD, Hulse, MA, Wilkinson, C, Hodson, A, and Gibson, M. The association football medical research programme: An audit of injuries in professional football. Br J Sports Med 35: 43-47, 2001.

16. Hooper, SL and Mackinnon, LT. Monitoring overtraining in athletes. Recommendations. Sports Med 20: 321-327, 1995.

17. Hopkins, WG. A scale of magnitudes for effect statistics. In: A New View of Statistics, 2011. Available at: http://sportsci.org/resource/ stats/index.html. Accessed 20 May, 2013.

18. Hopkins, WG. Precision of measurement. In: A New View of Statistics, 2011. Available at: http://newstats.org/precision.html. Accessed 20 May, 2013.

19. Howatson, $G$ and Milak, A. Exercise-induced muscle damage following a bout of sport specific repeated sprints. J Strength Cond Res 23: 2419-2424, 2009.

20. Ispirlidis, I, Fatouros, IG, Jamurtas, AZ, Nikolaidis, MG, Michailidis, I, Douroudos, I, Margonis, K, Chatzinikolaou, A, Kalistratos, E, Katrabasas, I, Alexiou, V, and Taxildaris, K. Timecourse of changes in inflammatory and performance responses following a soccer game. Clin J Sport Med 18: 423-431, 2008.

21. Kenttä, G and Hassmén, P. Overtraining and recovery. A conceptual model. Sports Med 26: 1-16, 1998.

22. Kinugasa, Tand Kilding, AE. A comparison of post-match recovery strategies in youth soccer players. J Strength Cond Res 23: 14021407, 2009.

23. Krustrup, P and Bangsbo, J. Physiological demands of top-class soccer refereeing in relation to physical capacity: Effect of intense intermittent exercise training. J Sports Sci 19: 881-891, 2001.

24. Magal, M, Dumke, CL, Urbiztondo, ZG, Cavill, MJ, Triplett, NT, Quindry, JC, McBride, JM, and Epstein, Y. Relationship between serum creatine kinase activity following exercise-induced muscle damage and muscle fibre composition. J Sports Sci 28: 257-266, 2010.

25. Magalhães, J, Rebelo, A, Oliveira, E, Silva, JR, Marques, F, and Ascensão, A. Impact of Loughborough Intermittent Shuttle Test versus soccer match on physiological, biochemical and neuromuscular parameters. Eur J Appl Physiol 108: 39-48, 2010.

26. McLean, BD, Coutts, AJ, Kelly, V, McGuigan, MR, and Cormack, SJ. Neuromuscular, endocrine, and perceptual fatigue responses during different length between-match microcycles in professional rugby league players. Int J Sports Physiol Perform 5:367383,2010

27. McLellan, CP and Lovell, DI. Neuromuscular responses to impact and collision during elite rugby league match play. J Strength Cond Res 26: 1431-1440, 2012.

28. Nicol, C, Avela, J, and Komi, PV. The stretch-shortening cycle: A model to study naturally occurring neuromuscular fatigue. Sports Med 36: 977-999, 2006.

29. Osgnach, C, Poser, S, Bernardini, R, Rinaldo, R, and di Prampero, PE. Energy cost and metabolic power in elite soccer: A new match analysis approach. Med Sci Sports Exerc 42: 170-178, 2010.

30. Peeling, P, Fulton, S, Sim, M, and White, J. Recovery effects of hyperoxic gas inhalation or contrast water immersion on the postexercise cytokine response, perceptual recovery, and next day exercise performance. J Strength Cond Res 26: 968-975, 2012.

31. Rahnama, N, Reilly, T, and Lees, A. Injury risk associated with playing actions during competitive soccer. Br J Sports Med 36: 354 359, 2002.

32. Rampinini, E, Bosio, A, Ferraresi, I, Petruolo, A, Morelli, A, and Sassi, A. Match-related fatigue in soccer players. Med Sci Sports Exerc 43: 2161-2170, 2011.

33. Ronglan, LT, Raastad, T, and Børgesen, A. Neuromuscular fatigue and recovery in elite female handball players. Scand J Med Sci Sports 16: 267-273, 2006.

34. Schache, AG, Crossley, KM, Macindoe, IG, Fahrner, BB, and Pandy, MG. Can a clinical test of hamstring strength identify football players at risk of hamstring strain? Knee Surg Sports Traumatol Arthrosc 19: 38-41, 2011.

35. Slattery, KM, Wallace, LK, Bentley, DJ, and Coutts, AJ. Effect of training load on simulated team sport match performance. Appl Physiol Nutr Metab 37: 315-322, 2012.

36. Small, K, McNaughton, L, Greig, M, and Lovell, R. The effects of multidirectional soccer-specific fatigue on markers of hamstring injury risk. J Sci Med Sport 13: 120-125, 2010.

37. Stølen, T, Chamari, K, Castagna, C, and Wisløff, U. Physiology of soccer: An update. Sports Med 35: 501-536, 2005.

38. Thorlund, JB, Aagaard, P, and Madsen, K. Rapid muscle force capacity changes after soccer match play. Int J Sports Med 30: 273-278, 2009

39. Young, WB, Hepner, J, and Robbins, DW. Movement demands in Australian rules football as indicators of muscle damage. J Strength Cond Res 26: 492-496, 2012. 